\title{
Calculating the Risk of Mountain Pine Beetle Attack: a Comparison of Distance- and Density-Based Estimates of Beetle Pressure
}

\author{
M. A. Wulder ${ }^{1 *}$, J. C. White ${ }^{1}$, C. C. Dymond ${ }^{1}$, T. Nelson ${ }^{2}$, B. Boots ${ }^{3}$ and T. L. Shore ${ }^{1}$ \\ ${ }^{1}$ Canadian Forest Service (Pacific Forestry Centre), Natural Resources Canada, Victoria, BC V8Z 1M5, Canada \\ ${ }^{2}$ University of Victoria, Department of Geography, Victoria, BC V8W 2Y2, Canada \\ ${ }^{3}$ Wilfrid Laurier University, Department of Geography and Environmental Studies, Waterloo, ON N2L 3C5, Canada
}

\begin{abstract}
An established model for risk rating of Pinus contorta stands for potential mortality caused by mountain pine beetle (Dendroctonus ponderosae) combines information on stand susceptibility and beetle pressure. Susceptibility is determined using attributes in the forest inventory data, while beetle pressure is calculated based on the size and distance to existing infestation locations (distance-based model). An alternate model for calculating beetle pressure is presented in this paper, which uses Voronoi polygons to incorporate size and distance, while emphasizing the density of existing infestation locations (density-based model), in combination with empirical knowledge of beetle dispersal and forest inventory data. Survey data of existing beetle damage were collected using a helicopter mounted global positioning system (GPS) at a study site in central British Columbia, Canada in 1999, 2000 , and 2001. These data facilitated the estimation of beetle pressure, and the comparison of risk ratings to actual attack locations. Using the distance-based model, 18 and $27 \%$ of areas identified as having a risk rating of greater than 50 in 1999 and 2000 were actually found to be attacked by beetles in surveys conducted in 2000 and 2001. Conversely, 39 and $49 \%$ of areas identified as having risk greater than 50 in 1999 and 2000 with the density-based model were attacked in 2000 and 2001. The results suggest that the density-based model of beetle pressure produced risk ratings that had a greater correspondence with actual infestation occurrence than risk ratings generated from the distance-based model. Using data that is typically collected to monitor beetle populations, novel methods of spatial processing may be applied in a transparent manner, generating results that incorporate knowledge of mountain pine beetle dynamics under certain population conditions, into calculations of the risk of mountain pine beetle attack.
\end{abstract}

Keywords: Beetle pressure, forest inventory, GPS, modelling, mountain pine beetle, risk, susceptibility, Voronoi diagram

\section{Introduction}

The mountain pine beetle (Dendroctonus ponderosae) is the most significant agent of mortality in mature lodgepole pine (Pinus contorta) forests in western North America (Furniss and Carolin, 1977). The total area impacted by mountain pine beetle in the province of British Columbia, Canada, has increased from approximately 164,000 ha in 1999 to 8.5 million hectares in 2005 (Westfall, 2006). The two main factors that have contributed to the successful expansion of the beetle population in British Columbia include the large amount of mature lodgepole pine on the land base, which has tripled in the last century as a result of intensive fire suppression activities (Taylor and Carroll, 2004) and several successive years of favourable climatic conditions, resulting in an increase in climatically suitable areas for brood development (Carroll et al., 2004).

Adult beetles typically attack a host tree in August and lay eggs, which complete their development cycle into mature adults approximately one year later (Amman and Cole, 1983). A mass-attack by mountain pine beetle leads to tree mortality through a combination of gallery excavation and the intro-

\footnotetext{
* Corresponding author: mwulder@nrcan.gc.ca
}

duction of fungal pathogens (Safranyik et al., 1974). Once killed, but still with green foliage, the host tree is in the green attack stage. The foliage of the host tree changes colour gradually; twelve-months after being attacked, over $90 \%$ of the killed trees will have red needles (red attack) (Amman, 1982; Henigman et al., 1999). Three years after being attacked, most trees will have lost all needles (grey attack) (British Columbia Ministry of Forests, 1995). Safranyik and Carroll (2006) provide a comprehensive synthesis of our current understanding of mountain pine beetle biology.

Identifying forest stands with the greatest risk for timber losses as a result of mountain pine beetle attack is critical information for mitigation and forest management planning. Decision support systems that incorporate the movement of pests and disease allow managers to fight outbreaks of these phenomena more efficiently (e.g. Hawksworth et al., 1995). These systems rely on quality data sources that are suitable for informing decision making. The dispersal and population density of pests are key elements of evaluating risk; however, landscape-scale movement of pests and diseases can be difficult to measure and predict (Liu et al., 2006). The relative importance of source population density and spread distances to the evaluation of risk associated with mountain pine beetle attack has not been investigated. Rather, risk models typically 
rely on factors such as stand characteristics and climatic suitability (Bentz et al., 1993). The susceptibility rating system is used by forest managers to predict the likelihood of attack and damage by mountain pine beetle. Several such systems, which use a wide range of different attributes to assess the risk of mountain pine beetle attack, have been developed in the last thirty years (Amman et al., 1977; Mahoney, 1978; Berryman, 1978; Schenk et al., 1980; Waring and Pitman, 1980; Stuart, 1984; Anhold and Jenkins, 1987). Shore et al. (2006) provide details and review the merits of these various rating systems.

In the Shore and Safranyik (1992) rating system, susceptibility is a product of four factors: stand age, stand density, percentage of susceptible pine basal area in the stand, and stand location. The susceptibility ratings do not include information on the presence of the existing mountain pine beetle populations (Shore et al., 2000). Therefore, a stand may have a high susceptibility to attack, but if there are no mountain pine beetles in the vicinity, the stand will not typically be at risk. Conversely, a low susceptibility to mountain pine beetle does not necessarily indicate a low likelihood of attack under epidemic population conditions (Shore and Safranyik, 1992; Bentz et al., 1993); the large size of mountain pine beetle populations during an epidemic phase and the resulting increaseed competition for suitable hosts could result in the beetles attacking sub-optimal host trees that would otherwise not be targeted, given different population conditions (Safranyik and Carroll, 2006). Since susceptibility is based on stand variables, it is typically a static rating that changes gradually, as the stand structure changes over time.

A risk rating system combines susceptibility with the location and size of nearby mountain pine beetle populations in order to characterize "the short-term expectancy of tree mortality in a stand as a result of the mountain pine beetle infestation" (Shore and Safranyik, 1992); or "the likelihood of an outbreak population occurring in a stand and concomitant loss during a specified period of time" (Bentz et al., 1993). The location and size of nearby beetle populations is referred to as beetle pressure. From an operational perspective, areas identified as having a high risk rating can subsequently be assigned priority for sanitation harvesting or other mitigation activities. Beetle pressure and risk ratings are dynamic indices that can change rapidly as a result of climatic factors or beetle dispersal, and must therefore be recalculated annually as new information on infestation conditions are collected (Shore and Safranyik, 1992).

Knowledge of beetle pressure is an important component in assessing risks of short-term losses in volume resulting from mountain pine beetle attack. The objective of this study is to compare two different approaches for calculating beetle pressure, and assess the impact these approaches have on the subsequent calculation of risk ratings. To meet this objective, beetle pressure was calculated using a traditional distancebased model, as well as an alternative density-based model, implemented with a Voronoi tessellation generated from the helicopter GPS point survey data of known beetle infestation locations. "Given a finite set of distinct, isolated points in a continuous space, we associate all locations in that space with the closest member of the point set. The result is a partitioning of the space into a set of regions." (Okabe et al., 2000). The Voronoi regions or polygons partition the space in such a way that each Voronoi polygon consists of an area that is closer to a given point than any other point. The Voronoi polygons have been used for point pattern analysis in a wide range of disciplines (Boots and Getis, 1988). There is an inverse proportional relationship between the size of the Voronoi polygons and the density of mountain pine beetle attack (as represented by the helicopter GPS survey data). The representation of the point density facilitated by the Voronoi polygons is intuitive: the higher the density of the survey points, the smaller the Voronoi polygons. Conversely, where survey points are sparse, Voronoi polygons are larger (Figure 1).

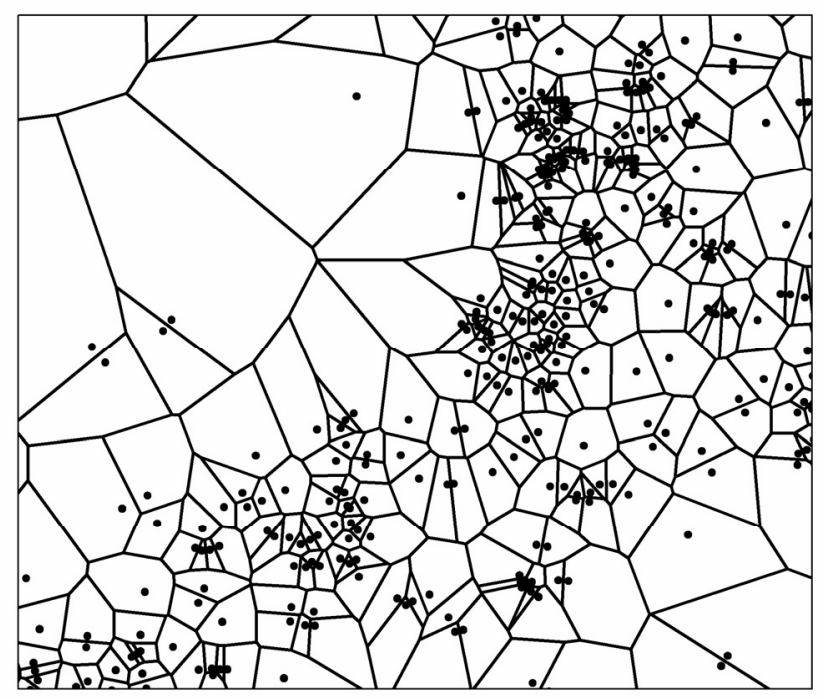

Notes: the space is represented by a greater number and smaller size of Voronoi polygons where the survey points are dense; conversely, the space is represented by fewer and larger Voronoi polygons where the survey points are less dense.

Figure 1. A sample of the Voronoi tessellation generated from the helicopter GPS survey data of known mountain pine beetle infestation locations.

Risk ratings were calculated for the study area by using the susceptibility combined with each form of beetle pressure, and these ratings were subsequently compared to survey data of known mountain pine beetle attack locations. Variations in risk ratings resulting from the methods used to estimate beetle pressure were then assessed and analyzed.

\section{Study Area and Data}

\subsection{Study Area}

The study area focuses on the southern portion of the Fort St. James Forest District in central British Columbia, Canada (Figure 2), representing approximately 10,000 square kilometres. The undulating topography ranges in elevation 


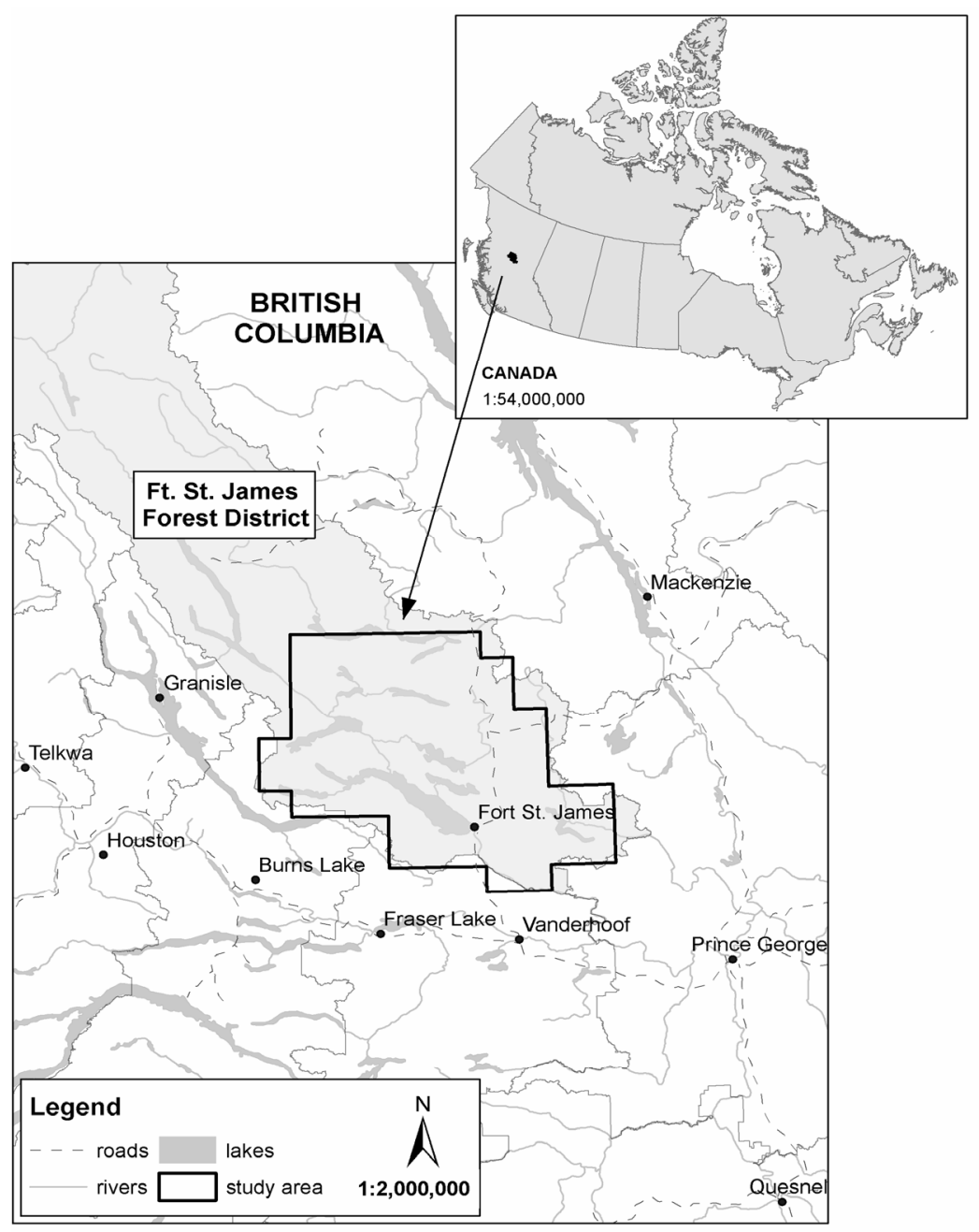

Figure 2. The study area is located in the southern portion of the Ft. St. James Forest District in central British Columbia, Canada.

from 712 to $1,394 \mathrm{~m}$ above the sea level and is dominated by several large fresh water lakes. This area is predominantly in the Sub-Boreal Spruce biogeoclimatic zone (Meidinger and Pojar, 1991). The climate of the region is continental and typically characterized by cold, snowy winters with short, warm, moist summers. There is moderate precipitation, 440 to 900 $\mathrm{mm}$ each year, with 25 to $50 \%$ of the precipitation in the form of snow.

The productive forest is dominated by lodgepole pine and white spruce (Picea glauca) with trembling aspen (Populous tremuloides), black spruce (Picea mariana) and Douglas-fir (Pseudotsuga menziezii) as the secondary species. A smaller amount of western red cedar (Thuja plicata) and sub-alpine fir (Abies lasiocarpa) is also found in this area. Pure and mixed stands of lodgepole pine cover most of the study region. Stands of aspen and Douglas-fir are found more frequently along the western margins of the region, while sub-alpine fir being found at higher elevations. Wildfires have left a mosaic of forest ages; lodgepole pine stands vary in age from less than 20 to more than 250 years.

One of the epicentres of current province-wide outbreak of mountain pine beetle was located near Ft. St. James (Figure 2 ). Forest harvesting is the primary land use activity in this area. In an effort to mitigate damage caused by the mountain pine beetle, the annual allowable cut in the area was initially increased by $30 \%$ in 2002 (Pedersen, 2002), followed by an additional 23\% increase in 2004 (Pedersen, 2004). The proportion of pine volume harvested in this area has increased from 50\% in 1998 (pre-epidemic) to 63\% in 2005 (Pousette and Hawkins, 2006).

\subsection{Forest Inventory}

Polygon based forest-inventory data are often utilized as information sources for decision support systems (Shore and Safranyik, 1992). The inventory is the primary source of information on the distribution and areal extent of the forest 
stands, logging roads, and past natural and human disturbances in the study region. Much of the information collected in the forest inventory is generated by interpretation of the aerial photographs, with field visits taken to ensure the quality and consistency of the interpretation. The inventory includes species composition (of up to six species, with estimates of species prevalence to the nearest $10 \%$ ), stand age in years, crown closure (to the nearest 5\%), stand height in metres, diameter at breast height in centimetres, and stand area in hectares (Gillis and Leckie, 1993). The information in the forest inventory represents a given point in time, and the temporal cycle for the inventory updates can vary (Gillis and Leckie, 1996). As a result, disturbance events such as harvesting or insect outbreaks may not be captured in the forest inventory until an update is completed. In the interim, data on disturbance events is typically captured, stored, and maintained separately from the forest inventory. The forest inventory of the study area is part of the Forest Inventory Planning (FIP) database maintained by the British Columbia Ministry of Forests, and represents forest conditions in 1999. The forest inventory data were used in the calculation of susceptibility ratings.

\subsection{Mountain Pine Beetle Survey Data}

A hierarchy of different data sources is used to detect and map the location and extent of damage caused by mountain pine beetle infestations (Wulder et al., 2006a). Field surveys are generally conducted during the green-attack stage, when there is no visual indication of damage in the foliage, but there are symptoms of attack in the lower bole and under the bark (Safranyik and Carroll, 2006). Aerial surveys are generally conducted to detect red attack damage, which is often readily discernible through visual inspection (Wulder et al., 2006b). Surveys conducted using a helicopter mounted global positioning system (GPS) record the location and magnitude of beetle damage. The helicopter GPS surveys capture clusters of trees with red attack damage, and the GPS is used to record the location of the cluster centroids. For each cluster, the number of infested trees is estimated and the species of infestation recorded. The maximum area represented by a point is typically 3 ha, equivalent to a circle with a radius of $100 \mathrm{~m}$ (Nelson et al., 2006). While a single point is used to identify a cluster of trees, the total area, size, shape, and compactness of clusters will vary and may depend on the surveyor.

The accuracy of the helicopter GPS surveys may be influenced by operational factors such as weather conditions, surveyor experience, and speed or flying height of the helicopter. Helicopter movement, shadow, view angle, and weather conditions may result in either overestimates or underestimates of attack severity and position (Leckie et al., 2005; Wulder et al., 2006a). Helicopter GPS surveys are considered the benchmark for operational accuracy in the detection and mapping of mountain pine beetle impacts at the local scale, with a horizontal positional accuracy of approximately $20 \mathrm{~m}$ and a low error of commission $(<5 \%$ ) (British Columbia Ministry of Forests, 2004). Research has examined the uncertainty and error associated with the point estimates derived from the heli-GPS surveys and has indicated that errors associated with the heli-GPS points (as determined by direct comparison to the ground surveys) were small; when estimating the numbers of attacked trees, $92.6 \%$ of heli-GPS points have errors of \pm 10 trees (Nelson et al., 2006). Information from the helicopter GPS surveys conducted in the study area in 1999 and 2000 were used to generate estimates of beetle pressure, while survey data collected in 2000 and 2001 were used to assess the variation in risk ratings (generated from the previous year's survey data) resulting from the different methods of estimating beetle pressure.

\section{Methods}

The Shore and Safranyik (1992) risk index is a function of both stand susceptibility and beetle pressure. Of the two components required to rate stand risk components, susceptibility relates to the level of damage that may occur when the stand experiences a mountain pine beetle infestation, while beetle pressure refers to the probability that the mountain pine beetle will enter the stand.

\subsection{Susceptibility}

Susceptibility to mountain pine beetle attack $(S)$ was calculated as per (Shore and Safranyik, 1992):

$S=P \times A \times D \times L$

where $P$ represents susceptible pine basal area, $A$ is the age of dominant and co-dominant live pine, $D$ is stand density, and $L$ is a location factor based on latitude, longitude, and elevation. Index values for $A, D$, and $L$, ranging from 0 to 1 , are generated and multiplied with $\mathrm{P}$ to produce a measure of susceptibility that ranges from 0 to 100 (Shore and Safranyik, 1992).

Some modifications were made to susceptibility rating calculations to enhance the consistency of the index values amongst stands. Continuous functions, rather than discrete classes, were used to calculate age, density, and location, in order to minimize abrupt differences in risk ratings caused by shifting from one discrete class to another, and to reflect developments to the model that have occurred since initial publication (Shore et al., 2006). The susceptibility model was also adapted to use the variables available in the FIP data (after Howse, 1995). Specifically, the FIP data does not contain the basal area of different species, or the density (stems per hectare), which were required by the Shore and Safranyik model. The FIP adaptation replaced the percent of pine by basal area with the percent of pine by stand volume, and stand density was replaced using the quadratic mean stand diameter (breast height) as a surrogate. In addition to the forest characteristics captured in the FIP database, the latitude and longitude (in decimal degrees), and the elevation (in meters) were obtained for each stand from a $25 \mathrm{~m}$ digital elevation model (British Columbia Ministry of Sustainable Resource Management, 2002). 


\subsection{Beetle Pressure Using Distance-based Model}

Beetle pressure was calculated for each stand based on helicopter-GPS survey data collected in the study area in 1999 and 2000. Beetle pressure is a measure of the size and proximity of the mountain pine beetle population influencing the stand being rated. Infestations are categorized as small, medium or large based on the size of the beetle population (Table 1). For each size category, distance to the nearest infestation is then used to compute a beetle pressure value. Thresholds used for generating beetle pressure are based on qualitative expert observations made during population and dispersal studies (Shore and Safranyik, 1992).

The Shore and Safranyik (1992) model for calculating beetle pressure is based on the number of infested trees inside the stand, the number of infested trees outside but within $3 \mathrm{~km}$ of the stand, and the distance between the stand being rated and the closest infestation. For example, a stand that contains between 1 and 10 infested trees, and has 900 to 9,000 infested trees within a $3 \mathrm{~km}$ radius, has a beetle pressure index of 0.8 . If instead, that stand contained no infested trees, and the closest infestation was between 1 and $2 \mathrm{~km}$ away, the beetle pressure index was 0.6 (Figure 3). The threshold values used to calculate beetle pressure are based on the results of population and dispersal studies (e.g. Safranyik, 1969; Safranyik et al., 1989; Shore and Safranyik et al., 1992).

\subsection{Beetle Pressure Using Density-based Model}

As an alternative to the distance-based model of beetle pressure, a density-based model was developed using Voronoi polygons (see Figure 3). Given a set of points in a continuous space, Voronoi polygons partition the space in such a way that each Voronoi polygon consists of an area that is closer to a given point than any other point (Okabe et al., 2000). Survey data of known locations of beetle infestation were used to generate the Voronoi polygons using the THIESSEN command in the Arc/Info ${ }^{\mathrm{TM}}$ geographic information system (GIS) software. As mentioned previously, there is an inverse proportional relationship between attack density and the size of the Voronoi polygons. These polygons of beetle density were then intersected with the forest inventory polygons, and when considered in conjunction with knowledge of beetle dispersal in epidemic population conditions, provided an indication of density-based beetle pressure for each forest stand.

Using the density-based model, beetle pressure was estimated based on the number of infested trees inside the stand, within $3 \mathrm{~km}$ of the stand, and the area of the Voronoi polygon overlapping the stand. The latter is unique to the densitybased model. For example, a stand that contained between 1 and 10 infested trees, with 900 to 9,000 infested trees within 3 $\mathrm{km}$ had a beetle pressure index of 0.8 ; the same as in the distance-based model (Table 1). If instead the stand contained no infested trees, and the area of the Voronoi polygon, which intersected the stand being rated, was between 30 and 60 ha, the beetle pressure index would be 0.6 (Table 1). The 30 ha and 60 ha thresholds were developed based on spatial simulations of beetle populations and empirical mark-recapture studies. Logan et al. (1998) demonstrate that when the source areas of beetles occur in high density (1 source cluster per 15 to 80 hectares, depending on population size), spatial patterns of attacked trees replicate the clustering pattern seen in incipient and epidemic infestations (Logan et al., 1998). Several experimental studies show that after release, 75 to $95 \%$ of the beetles recaptured are within a 30 ha area (300 m radius), and 90 to $95 \%$ are recaptured within a 60 ha area (between 400 to $500 \mathrm{~m}$ radius), although the percent recaptured depends on the experimental design (Safranyik et al., 1992; Turchin and Thoeny, 1993; Byers, 1999).

\subsection{Stand Risk}

Risk $(R)$ was calculated by combining the susceptibility $(S)$ and the beetle pressure $(B)$ components, as per Shore and Safranyik (1992):

Table 1. Mountain Pine Beetle Intensity (A) and Beetle Pressure (B)

(A) Beetle Intensity

\begin{tabular}{llll}
\hline \multirow{2}{*}{$\begin{array}{l}\text { Number of infested trees } \\
\text { outside stand within } 3 \mathrm{~km}\end{array}$} & \multicolumn{4}{l}{ Number of infested trees inside the stand } \\
\cline { 2 - 4 } & $<10$ & $10-100$ & $>100$ \\
\hline$<900$ & Small & Medium & Large \\
900 to 9,000 & Medium & Medium & Large \\
$>9,000$ & Large & Large & Large \\
\hline
\end{tabular}

(B) Beetle Pressure

\begin{tabular}{llllllllll}
\hline \multirow{2}{*}{$\begin{array}{l}\text { Relative intensity } \\
\text { of infestation }\end{array}$} & $\begin{array}{l}\text { Within } \\
\text { stand }\end{array}$ & \multicolumn{3}{l}{ Distance to nearest infestation $(\mathrm{km})$} & \multicolumn{4}{c}{ Area of overlapping Voronoi polygon (ha) } \\
\cline { 3 - 11 } & $0-1$ & $1-2$ & $2-3$ & $3-4$ & $>4$ & $0-30$ & $30-60$ & $>60$ \\
\hline Small & 0.6 & 0.5 & 0.4 & 0.3 & 0.1 & 0.06 & 0.5 & 0.4 & 0.06 \\
Medium & 0.8 & 0.7 & 0.6 & 0.4 & 0.2 & 0.08 & 0.7 & 0.6 & 0.08 \\
Large & 1.0 & 0.9 & 0.7 & 0.5 & 0.2 & 0.1 & 0.9 & 0.7 & 0.1 \\
\hline
\end{tabular}




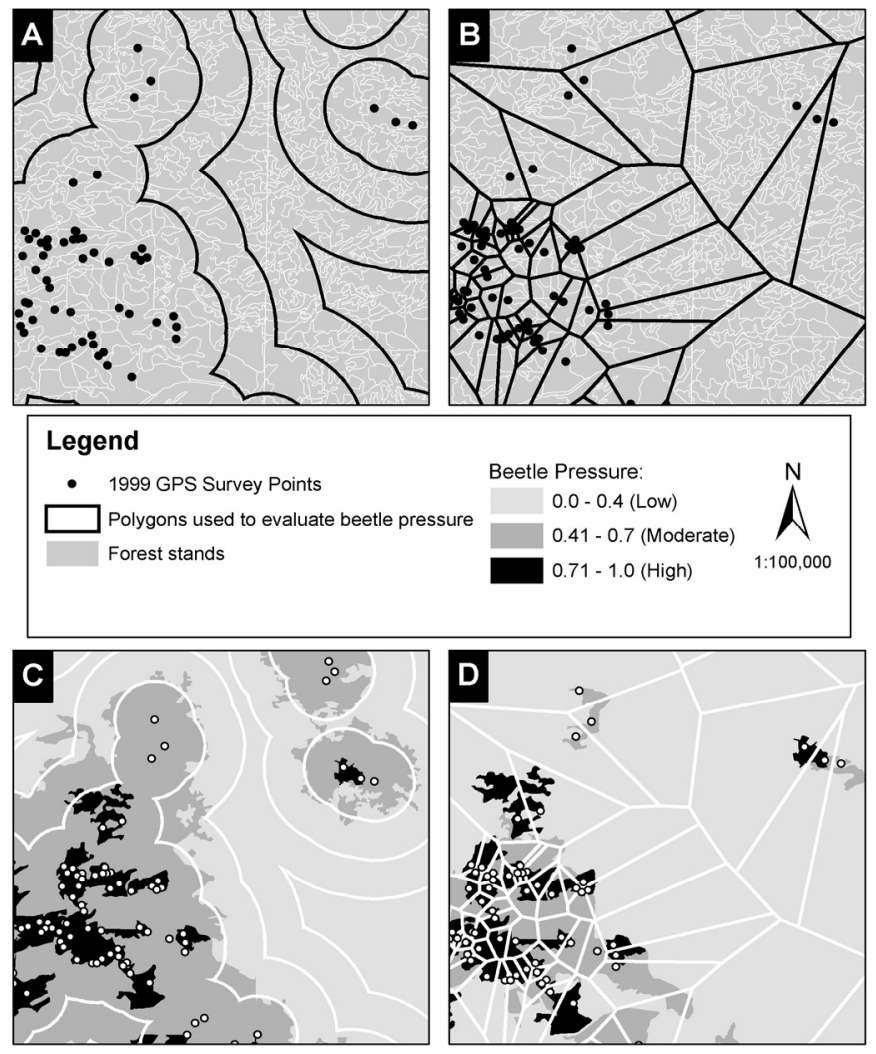

Notes: The distance-based model was generated by $1 \mathrm{~km}$ buffers applied to the helicopter GPS survey points; the density-based model was generated using Voronoi polygons; the resulting beetle pressure values are shown in (C) distance-based model and (D) density-based models.

Figure 3. Examples illustrating the calculation of (A) distance-based and (B) density-based models of beetle pressure.

$$
R=2.74\left(S^{1.77} e^{-0.0177 S}\right)\left(B^{2.78} e^{-2.78 B}\right)
$$

Risk was modeled as the short-term probability of loss of stand basal area. High risk does not indicate that a particular stand will be attacked; rather, the stand is considered to be at greater risk than stands with lower risk ratings. Risk ratings range from 0 to 100 .

\subsection{Correspondence to MPB Survey Data}

In order to assess the variation in beetle pressure and risk ratings generated from the distance and density approaches, the ratings for 1999 and 2000 were compared to actual locations of mountain pine beetle attack in 2000 and 2001 respectively using GIS software. The forest inventory stand boundaries, with their estimated beetle pressure/risk ratings were intersected with the heli-GPS survey locations. If a heli-GPS survey point was found within a forest inventory stand, the stand was considered to have been attacked by mountain pine beetle. Conversely, if no survey points were found within the stand, the stand was considered to not have been attacked.
The mountain pine beetle helicopter GPS survey data is described in Section 2.3.

\section{Results}

Of the forest stands in the study area with some level of susceptibility, the greatest proportion (28\%) had a susceptibility rating less than 10 (Table 2). Stands with a low susceptibility rating (e.g. < 20) were dominated by spruce or small diameter pine. Conversely, stands with a high susceptibility rating (e.g. $>80$ ) were dominated by large diameter lodgepole pine. Only a small number of forest stands in the study area had high beetle pressure ratings (e.g. $\geq 0.7$ ), for either the distance- or density-based models, in 1999 or 2000 (Tables 3 and 4). The greatest differences between the two models of estimating beetle pressure are found in the low and moderate beetle pressure classes. The distance-based model for calculating beetle pressure resulted in a greater number of stands with moderate beetle pressure (Table 3 ), whereas the densitybased model resulted in more stands with low beetle pressure (Table 4). 
Table 2. Distribution of Forest Stands by Susceptibility Rating

\begin{tabular}{ll}
\hline Susceptibility Rating & Number of Forest Stands \\
\hline 0 & 30,681 \\
$1-10$ & 9,558 \\
$11-20$ & 3,911 \\
$21-30$ & 3,083 \\
$31-40$ & 2,640 \\
$41-50$ & 2,308 \\
$51-60$ & 3,193 \\
$61-70$ & 2,488 \\
$71-80$ & 3,279 \\
$81-90$ & 2,167 \\
$91-100$ & 1,227 \\
\hline
\end{tabular}

Table 3. Distribution of Forest Stands, by Distance-based Estimates of Beetle Pressure

\begin{tabular}{lll}
\hline Beetle pressure & 1999 & 2000 \\
\hline High $(\geq 0.7)$ & 2,332 & 2,917 \\
Moderate $(0.4-0.7)$ & 29,939 & 27,333 \\
Low $(\leq 0.4)$ & 32,323 & 34,344 \\
\hline
\end{tabular}

Table 4. Distribution of Forest Stands, by Density-based Estimates of Beetle Pressure

\begin{tabular}{lll}
\hline Beetle pressure & 1999 & 2000 \\
\hline High $(\geq 0.7)$ & 1,494 & 1,730 \\
Moderate $(0.4-0.7)$ & 3,759 & 4,228 \\
Low $(\geq 0.4)$ & 59,341 & 58,636 \\
\hline
\end{tabular}

Results from the distance-based model showed that actual mountain pine beetle infestations occurred in stands with high, moderate, and low beetle pressure values (Table 5). However, as the beetle pressure increased, the proportion of forest stands with infestations also increased. For example, in $2000,39.5 \%$ of stands with high beetle pressure in 1999 were attacked, whereas only $1.5 \%$ of stands with low beetle pressure values were attacked. Similarly in 2001, 31.2\% of stands with high beetle pressure values were attacked in 2000, while only $4.8 \%$ of stands with low beetle pressure were attacked.

A comparable trend was found for the density-based model of beetle pressure (Table 6). In 2000, 55.1\% of stands with high beetle pressure in 1999 were attacked, while only $3.3 \%$ of stands with low beetle pressure were attacked. Similarly in 2001, 47.4 and $7.7 \%$ of stands with high and low beetle pressure values in 2000 , respectively, were attacked. The correspondence between the actual attack and high beetle pressure was greater when the density-based model was used; however, a slightly higher rate of attack also occurred in the stands with the lowest values of beetle pressure.
Table 5. Distance-based Model of Beetle Pressure: Number of Stands in Each Class of Beetle Pressure and Infestation Status in the Subsequent Year

\begin{tabular}{lll}
\hline $\begin{array}{l}\text { Beetle pressure from } \\
1999\end{array}$ & $\begin{array}{l}\text { Stands with } \\
\text { attack in 2000 }\end{array}$ & $\begin{array}{l}\text { Stands without attack } \\
\text { in 2000 }\end{array}$ \\
\hline High $(\geq 0.7)$ & $920(39.5 \%)$ & $1,412(60.5 \%)$ \\
Moderate $(0.4-0.7)$ & $2,317(7.7 \%)$ & $27,622(92.3 \%)$ \\
Low $(\leq 0.4)$ & $480(1.5 \%)$ & $31,843(98.5 \%)$ \\
\hline Beetle pressure from & $\begin{array}{l}\text { Stands with } \\
\text { attack in } 2001\end{array}$ & Stands without attack \\
\hline High $(\geq 0.7)$ & $909(31.2 \%)$ & $2,008(68.8 \%)$ \\
Moderate $(0.4-0.7)$ & $4,170(15.3 \%)$ & $23,163(84.7 \%)$ \\
Low $(\leq 0.4)$ & $1,644(4.8 \%)$ & $32,701(95.2 \%)$ \\
\hline
\end{tabular}

Table 6. Density-based Model of Beetle Pressure: Number of Stands in Each Class of Beetle Pressure and Infestation Status in the Subsequent Year

\begin{tabular}{lll}
\hline $\begin{array}{l}\text { Beetle pressure from } \\
1999\end{array}$ & $\begin{array}{l}\text { Stands with } \\
\text { attack in 2000 }\end{array}$ & $\begin{array}{l}\text { Stands with no attack in } \\
2000\end{array}$ \\
\hline High $(\geq 0.7)$ & $823(55.1 \%)$ & $671(44.9 \%)$ \\
Moderate $(0.4-0.7)$ & $905(24.1 \%)$ & $2,854(76.9 \%)$ \\
Low $(\leq 0.4)$ & $1,989(3.3 \%)$ & $57,352(96.7 \%)$ \\
\hline Beetle pressure from & $\begin{array}{l}\text { Stands with } \\
\text { attack in } 2001\end{array}$ & Stands with no attack in \\
\hline High $(\geq 0.7)$ & $820(47.4 \%)$ & $910(52.6 \%)$ \\
Moderate $(0.4-0.7)$ & $1,393(32.9 \%)$ & $2,835(67.1 \%)$ \\
Low $(\leq 0.4)$ & $4,510(7.7 \%)$ & $54,127(92.3 \%)$ \\
\hline
\end{tabular}

An assessment of how the two methods for estimating beetle pressure influenced the correspondence between risk rating and actual attack by mountain pine beetle was then undertaken. Risk ratings for 1999 and 2000 were categorized into ten-unit intervals. The proportion of stands within each risk category that were attacked or not attacked was then tabulated (Table 7). The trends for both models of beetle pressure, for both years, are very similar: as the risk rating increases, the proportion of stands with that particular risk rating that were actually attacked, also increases. Furthermore, the proportion of stands with attack, for all risk ratings, was greater in 2001 than 2000. This is expected as the beetle population grows and the infestation increases in size.

While the trends between the two approaches for estimating risks are similar, the density-based model generated risk estimates that had a greater correspondence with actual attack. For example, if the risk ratings are further aggregated into risk less than 50 and risk greater than 50, 18\% and $27 \%$ of areas identified as having a risk rating of greater than 50 in 1999 and 2000 using the distance-based model were actually found to be attacked by beetles in surveys conducted in 2000 and 2001. Conversely, 39 and $49 \%$ of areas identified as having risk greater than 50 in 1999 and 2000 with the density- 


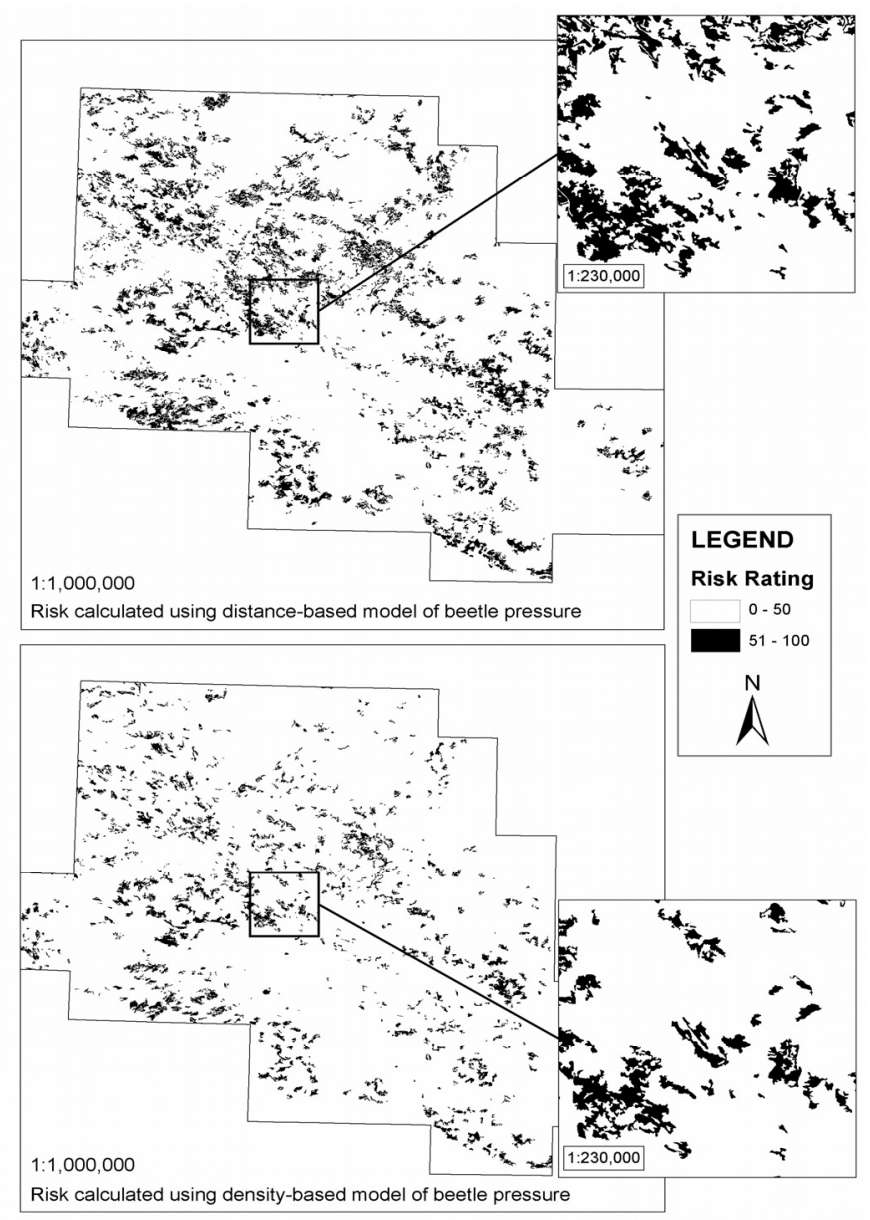

Figure 4. Risk ratings for 2000 generated using distance- and density-based models of beetle pressure.

based model were attacked in 2000 and 2001. The differences between the two approaches to estimating beetle pressure and the resulting differences in risk ratings are also apparent in Figures 3 and 4, which illustrates the spatial distribution of risk for 2000. The density-based model estimated twice as much area as having risk between 0 and 10 compared to the distance-based model $(477,263$ ha versus $232,711 \mathrm{ha})$, while the distance-based model estimated 53,810 ha with risk between 11 and 20, compared to the 9,925 ha with the density model.

\section{Discussions}

Beetle pressure is an important component for rating the risk of mountain pine beetle attack. Two different approaches for estimating the beetle pressure were tested for comparison. While both approaches accounted for dispersal distances and infestation size, the distance-based model emphasized the distance between the stand being evaluated and the nearest infestation, while the density-based model emphasized the density of infestation points around the stand being evaluated. Overall, the density-based model of beetle pressure, developed using the Voronoi polygons, generated high risk ratings that more closely corresponded with actual attack by the mountain pine beetles.

A number of factors may have confounded results where stands experiencing high beetle pressure (and which had a high risk rating) were not attacked in the subsequent year. It is difficult to predict the outcome of natural processes such as dispersal and population dynamics of mountain pine beetle. An additional factor in this study area may have been the depletion of resources (Safranyik et al., 1974), as the mountain pine beetle epidemic in the Ft. St. James Forest District started before the 1999 base year for this research (British Columbia Ministry of Forests, 2002). Forest harvesting, as a component of an insect mitigation strategy (salvage harvesting), would also act to reduce the number of possible insect hosts. In the case where a forest inventory is outdated, it is possible that susceptibility would be overestimated, minimizing sensitivity to beetle pressure, and resulting in an overesti- 
Table 7. Risk Rating: Correspondence between 1999 and 2000 Risk Ratings and Actual Attack by Mountain Pine Beetle in 2000 and 2001

A) Distance-Model

\begin{tabular}{lcccc}
\hline \multirow{2}{*}{$\begin{array}{l}\text { Risk } \\
\text { Rating }\end{array}$} & \multicolumn{2}{c}{ Using 1999 Risk Rating (\%) } & \multicolumn{2}{c}{ Using 2000 Risk Rating (\%) } \\
\cline { 2 - 5 } & Stands with attack in 2000 & Stands without attack in 2000 & Stands with attack in 2001 & Stands without attack in 2001 \\
\hline $1-10$ & 3.88 & 96.12 & 7.93 & 92.07 \\
$11-20$ & 7.81 & 92.19 & 14.54 & 85.46 \\
$21-30$ & 8.21 & 91.79 & 13.98 & 86.02 \\
$31-40$ & 9.61 & 90.39 & 15.90 & 84.10 \\
$41-50$ & 10.85 & 89.15 & 19.24 & 80.76 \\
$51-60$ & 13.17 & 86.83 & 21.98 & 78.02 \\
$61-70$ & 40.50 & 59.50 & 49.82 & 50.18 \\
$71-80$ & 36.38 & 63.62 & 49.13 & 50.87 \\
$81-90$ & 56.09 & 43.91 & 49.21 & 50.79 \\
$91-100$ & 58.29 & 41.71 & 72.05 & 27.95 \\
\hline
\end{tabular}

B) Density-Model

\begin{tabular}{lcccc}
\hline Risk & \multicolumn{2}{c}{ Using 1999 Risk Rating (\%) } & \multicolumn{2}{c}{ Using 2000 Risk Rating (\%) } \\
\cline { 2 - 5 } Rating & Stands with attack in 2000 & Stands without attack in 2000 & Stands with attack in 2001 & Stands without attack in 2001 \\
\hline $1-10$ & 5.54 & 94.46 & 10.92 & 89.08 \\
$11-20$ & 24.90 & 75.10 & 37.43 & 62.57 \\
$21-30$ & 30.42 & 69.58 & 34.44 & 65.56 \\
$31-40$ & 25.47 & 74.53 & 31.45 & 68.55 \\
$41-50$ & 25.06 & 74.94 & 37.56 & 62.44 \\
$51-60$ & 33.00 & 67.00 & 40.98 & 59.02 \\
$61-70$ & 41.22 & 58.78 & 50.37 & 49.63 \\
$71-80$ & 37.01 & 62.99 & 54.14 & 45.86 \\
$81-90$ & 62.72 & 37.28 & 54.65 & 45.35 \\
$91-100$ & 64.51 & 34.49 & 75.35 & 24.65 \\
\hline
\end{tabular}

mation of risk.

Similarly, there are several potential reasons why stands with low risk ratings were attacked. As was previously mentioned, in epidemic population conditions, when competition for suitable hosts increases, beetles will attack sub-optimal hosts (Safranyik and Carroll, 2006). Furthermore, since outbreak conditions existed in much of the region surrounding the study area in the years considered (1999 to 2002), beetles could have emigrated from other areas via some long range dispersal mechanism (Safranyik and Carroll, 2006). Such an influx would exacerbate beetle pressure. It should also be noted that the helicopter GPS surveys were not conducted in a systematic manner across the study area, and that no information was recorded for locations where there was no attack. Therefore, for the purposes of this analysis, an assumption was made that stands that did not contain any survey points were not attacked. Notwithstanding the potential for error associated with this assumption, the helicopter GPS survey data is the benchmark of mountain pine beetle survey information (Wulder et al., 2006b), and has generally been found to be accurate in detecting locations of red attack damage
(Nelson et al., 2006).

The calculation of beetle pressure depends on a number of assumptions, including: dispersal distances, dispersal directionality, and population density of the mountain pine beetle. Further research in these areas could reduce the uncertainty in the model output (Higgins et al., 2003). Dispersal distances have been measured and modeled below the forest canopy (Safranyik et al., 1992; Powell et al., 2000). However, a large percentage of the population remains unaccounted for, and little is known about dispersal above the canopy (Safranyik et al., 1992; Barclay et al., 1998). When calculating beetle pressure, the beetles are assumed to disperse equally in all directions; however, since they are largely moved passively by the wind, the dispersal may follow from the predominant wind direction (Byers, 2000). The interactions between population size, aggregation, disaggregation, and resource depletion are being researched and modeled at the local scale (Logan et al., 1998; Safranyik et al., 1999). A mountain pine beetle infestation represents a complex natural process, impacting large areas ecologically and economically. Ongoing research is attempting to improve the characterization of mountain pine 
beetle movement spatially and temporally at the landscape scale (Safranyik and Wilson, 2006).

Although there are comparable trends between the two risk rating outputs, the distance-based model of beetle pressure identified more stands with a higher risk rating than the density-based model. The Voronoi polygons used in the density-based model incorporated the density of the helicopter GPS survey points into the estimates of beetle pressure. When beetle populations are increasing, more areas of damage will be identified in the survey, increasing the density of the survey points and resulting in smaller Voronoi polygons, which in turn result in a more spatially constrained rating of risk. With the distance based model, the density of the survey points has no bearing on the beetle pressure. In an operational context, a forest manager will want to prioritize areas for mitigation as resources will typically be limited. Using the distance based model, a much larger area would require action. However, by incorporating knowledge of beetle population dynamics and accounting for the density of the survey points through the spatial analysis, a spatially refined rating of risk results. We hypothesize that the density-based model is effecttive when mountain pine beetle populations are increasing and the density of surveyed damage is also increasing. Therefore, under certain population conditions, it may be preferable to emphasize the density of, rather than the distance to, nearby beetle infestations. Further testing of the density-based model under a range of population conditions would be required to test this hypothesis.

\section{Conclusions}

Assessing the stand-level risk associated with mountain pine beetle attack is a useful tool for predicting where attack is most likely to occur. Such information may be used by forest managers to prioritize areas for mitigation efforts. Risk is a function of stand susceptibility and beetle pressure, with the latter typically calculated by considering the distance and the size of mountain pine beetle populations within a certain proximity to the stand being rated. An alternate approach to calculating beetle pressure that considers the size, distance, and density of beetle populations was presented in this paper. Voronoi polygons represent the density of attack by mountain pine beetle in an intuitive manner: the inverse proportional relationship between the size of the Voronoi polygon and the density of attack facilitates interpretation. Previous research has indicated that high density population source areas may increase the risk of attack, primarily as a result of the massive number of beetles dispersing across the landscape. Given this, density may be an alternate means to estimate beetle pressure under an epidemic population scenario, although the approach presented herein would require testing under a broader range of population and stand-level conditions prior to implementtation in an operational context. In our study area, risk ratings generated from the density based model of beetle pressure corresponded more closely with actual beetle attack locations in the subsequent year, when compared to risk ratings generated from a distance-based model.
Acknowledgments. This project is funded by the Government of Canada through the Mountain Pine Beetle Initiative, a six-year, $\$ 40$ million program administered by Natural Resources Canada, Canadian Forest Service. Additional information on the Mountain Pine Beetle Initiative may be found at: http://mpb.cfs.nrcan.gc.ca/. Tim Ebata, of the British Columbia Ministry of Forests and Range, is thanked for provision of GPS survey data.

\section{References}

Amman, G.D., McGregor, M.D., Cahill, D.B., Klein, W.H. (1977). Guidelines for Reducing Losses of Lodgepole Pine to the Mountain Pine Beetle in Unmanaged Stands in the Rocky Mountains, USDA Forest Service, Intermountain Forest and Range Experiment Station, General Technical Report INT-36, pp. 19.

Amman, G.D. (1982). The mountain pine beetle-identification, biology, causes of outbreaks, and entomological research needs, in D.M. Shrimpton (Ed.), Proc. of the Joint Canada/USA Workshop on Mountain Pine Beetle Related Problems in Western North America, Environment Canada, Canadian Forestry Service, Pacific Forest Research Centre, Victoria, BC, Canada, Information Report BC-X-230, pp. 87.

Amman, G.D. and Cole, W.E. (1983). Mountain Pine Beetle Dynamics in Lodgepole Pine Forests Part 2: Population Dynamics, United States Department of Agriculture Forest Service GTR-INT-145, pp. 59.

Anhold, J.A. and Jenkins, M.J. (1987). Potential mountain pine beetle (Coleoptera: Scolytidae) attack of lodgepole pine as described by stand index. Environ. Entomol., 16, 738-742.

Barclay, H.J., Safranyik, L. and Linton, D. (1998). Trapping mountain pine beetles Dendroctonus ponderosae (Coleoptera: Scolytidae) using pheromone-baited traps: Effects of trapping distance. J. Entomol. Soc. BC, 95, 25-31.

Bentz, B.J., Amman, G.D. and Logan, J.A. (1993). A critical assessment of risk classification systems for the mountain pine beetle. For. Ecol. Manage, 61, 349-366.

Berryman, A.A. (1978). A synoptic model of the lodgepole pine/ mountain pine beetle interaction and its potential application in forest management, in A.A. Berryman, G.D. Amman, R.W. Stark and D.L. Kibbee (Eds.), Proc. of Theory and Practice of Mountain Pine Beetle Management in Lodgepole Pine Forests, Pullman, WA, University of Idaho, Moscow, ID, pp. 224.

Boots, B.N. and Getis, A. (1988). Point Pattern Analysis, Scientific Geography Series, Volume 8, Newbury Park, Sage Publications, pp. 93.

British Columbia Ministry of Forests (1995). Bark beetle management guidebook (Forest Practices Code) Forest Practices Branch, Victoria, BC Canada, pp. 45. www.for.gov.BC.ca/tasb/legsregs/ $\mathrm{fpc} /$ fpcguide/beetle/betletoc.htm.

British Columbia Ministry of Forests (2002). Historical distribution of damage mountain pine beetle cumulative damage history from about 1959 to 2002. http://www.for.gov.bc.ca/hfp/forsite/ overview/mpb_history.htm.

British Columbia Ministry of Forests (2004). British Columbia ministry of forests land-base investment program: Aerial detection standards for bark beetle management, Interim for Fiscal Year 2004-2005. http://www.for.gov.bc.ca/hcp/fia/landbase/Aeri alDetectionStandardforBarkBeetleManagement.doc.

British Columbia Ministry of Sustainable Resource Management (2002). Gridded digital elevation model product specifications, Edition 2.0, Base Mapping and Geomatics Services Branch, Victoria, BC. http://ilmbwww.gov.bc.ca/bmgs/products/BC-DE M-specifications-2002-12.pdf.

Byers, J.A. (1999). Effects of attraction radius and flight paths on catch of Scolytid beetles dispersing outward through rings of 
pheromone traps. J. Chem. Ecol., 25, 985-1005.

Byers, J.A. (2000). Wind-aided dispersal of simulated bark beetles flying through forests. Ecol. Model., 125, 231-243.

Carroll, A.L., Taylor, S.W., Régnière, J. and Safranyik, L. (2004). Effects of climate change on range expansion by the mountain pine beetle in British Columbia, in T.L. Shore, J.E. Brooks, J.E. Stone (Eds.), Mountain Pine Beetle Symposium: Challenges and Solutions, Natural Resources Canada, Canadian Forest Service, Pacific Forestry Centre, Victoria, BC, Information Report BCX-399, pp. 298.

Furniss, R.L. and Carolin, V.M. (1977). Western Forest Insects, United States Department of Agriculture Forest Service Miscellaneous Publication Number 1339, pp. 654

Gillis, M.D. and Leckie, D.G. (1993). Forest inventory mapping procedures across Canada, Petawawa National Forestry Institute, Forestry Canada, Information Report PI-X-114, pp. 74.

Gillis, M.D and Leckie, D.G. (1996). Forest inventory update in Canada. The For. Chronicle, 72, 138-156.

Hawksworth, F.G., Williams-Cipriani, J.C., Eav, B.B., Geils, B.G., Johnson, R.R., Marsden, M.A., Beatty, J.S., Shubert, G.S. and Robinson, D.C.E. (1995). Dwarf Mistletoe Impact Modeling System User's Guide and Reference Manual, United States Department of Agriculture Forest Service Report MAG-95-2, pp. 120

Henigman, J., Ebata, T., Allen, E. and Pollard, A. (Eds.) (1999). Field Guide to Forest Damage in British Columbia, British Columbia Ministry of Forests, Victoria, BC, pp. 343.

Higgins, S.I., Clark, J.S., Nathan, R., Hovestadt, T., Schurr, F., Fragoso, J.M.V., Aguiar, M.R., Ribbens, E. and Lavorel, S. (2003). Forecasting plant migration rates: Managing uncertainty for risk assessment. J. Ecol., 91, 341-347.

Howse, K. (1995). Stand Susceptibility Rating System for Bark Beetles Using SAS, Caribou Forest Region, British Columbia Ministry of Forests, Internal document.

Leckie, D., Cloney, E. and Joyce, S. (2005). Automated detection and mapping of crown discolouration caused by jack pine budworm with $2.5 \mathrm{~m}$ resolution multispectral imagery. Int. J. Appl. Earth Obs. Geoinf., 7, 61-77.

Liu, D., Kelly, M. and Gong, P. (2006). A spatial-temporal approach to monitoring forest disease spread using multi-temporal high spatial resolution imagery. Remote Sens. Environ., 101, $167-$ 180.

Logan, J.A., White, P., Bentz, B.J. and Powell, J.A. (1998). Model analysis of spatial patterns in mountain pine beetle outbreaks. Theor. Popul. Biol., 53, 236-255.

Mahoney, R.L. (1978). Lodgepole pine/mountain pine beetle risk classification methods and their application, in A.A. Berryman, G.D. Amman, R.W. Stark and D.L. Kibbee (Eds.), Proc. of Theory and Practice of Mountain Pine Beetle Management in Lodgepole Pine Forests, Pullman, WA, University of Idaho, Moscow, ID, pp. 224.

Meidinger, D. and Pojar, J. (Eds.) (1991). Ecosystems of British Columbia, Research Branch, Ministry of Forests, Victoria, BC, Canada, pp. 330

Nelson, T., Boots, B. and Wulder, M.A. (2006). Large-area mountain pine beetle infestations: Spatial data representation and accuracy. The For. Chronicle, 82, 243-252.

Okabe, A., Boots, B., Sugihara, K. and Chiu, S.N. (2000). Spatial Tessellations: Concepts and Applications of Voronoi Diagrams, $2^{\text {nd }}$ Edition, John Wiley \& Sons, pp. 671.

Pedersen, L. (2002). Prince George Timber Supply Area: Rationale for Allowable Annual Cut (AAC) Determination, British Columbia Ministry of Forests, Victoria, BC, Canada, pp. 100

Pedersen, L. (2004). Prince George Timber Supply Area: Rationale for Allowable Annual Cut (AAC) Determination, British Columbia Ministry of Forests, Victoria, BC, Canada, pp. 63.
Pousette, J. and Hawkins, C. (2006). An assessment of critical assumptions supporting the timber supply modelling for mountain-pine-beetle-induced allowable annual cut uplift in the Prince George Timber Supply Area. BC J. Ecosystems and Management, 7, 93-104. http://www.forrex.org/publications/jem/IS S35/vol7 no2 art10.pdf.

Powell, J., Kennedy, B., White, P., Bentz, B., Logan, J. and Roberts, D. (2000). Mathematical elements of attack risk analysis for mountain pine beetles. J. Theor. Biol., 204, 601-620.

Safranyik, L. (1969). Development of a Technique for Sampling Mountain Pine Beetle Populations in Lodgepole Pine, Ph.D. Dissertation, Univeristy of British Columbia, Vancouver BC, pp. 195.

Safranyik, L., Shrimpton, D. and Whitney, H. (1974). Management of Lodgepole Pine to Reduce Losses from the Mountain Pine Beetle, Canadian Forest Service, Victoria, BC, Forestry Technical Report, pp. 23.

Safranyik, L., Silversides, R., McMullen, L.H. and Linton, D.A. (1989). An empirical approach to modeling the local dispersal of the mountain pine beetle (Dendroctonus ponderosae Hopk., Coleoptera: Scolytidae) in relation to sources of attraction, wind direction and speed. J. Appl. Entomol., 108, 498-511.

Safranyik, L., Linton, D.A., Silversides, R. and McMullen, L.H. (1992). Dispersal of released mountain pine beetles under the canopy of a mature lodgepole pine stand. J. Appl. Entomol., 113, 441-450.

Safranyik, L., Barclay, H., Thomson, A. and Riel, W.G. (1999). A Population Dynamics Model for the Mountain Pine Beetle, Dendroctonus ponderosae Hopk. (Coleoptera: Scolytidae), Canadian Forest Service, Victoria, BC, Information Report BCX-386, pp. 24.

Safranyik, L. and Carroll, A. (2006). The biology and epidemeology of the mountain pine beetle in lodgepole pine forests, in L. Safranyik and B. Wilson (Eds.), The Mountain Pine Beetle: A Synthesis of Biology, Management, and Impacts on Lodgepole Pine, Natural Resources Canada, Canadian Forest Service, Pacific Forestry Centre, Victoria, BC, Canada, pp. 304.

Safranyik, L. and Wilson, B. (2006). The Mountain Pine Beetle: A Synthesis of Biology, Management, and Impacts on Lodgepole Pine, Natural Resources Canada, Canadian Forest Service, Pacific Forestry Centre, Victoria, BC, Canada, pp. 304.

Schenk, J.L., Mahoney, R.L., Moore, J.A. and Adams, D.L. (1980). A model for hazard rating lodgepole pine stands for mortality by mountain pine beetle. For. Ecol. Manage., 3, 57-66.

Shore, T.L. and Safranyik, L. (1992). Susceptibility and Risk Rating Systems for the Mountain Pine Beetle in Lodgepole Pine Stands, Forestry Canada, Victoria, BC, Information Report BC-X-336, pp. 12.

Shore, T.L., Safranyik, L. and Lemieux, J.P. (2000). Susceptibility of lodgepole pine stands to the mountain pine beetle: testing of a rating system. Can. J. Forest Res., 30, 44-49.

Shore, T.L., Riel, B.G., Safranyik, L. and Fall, A. (2006). Decision support systems, in L. Safranyik and B. Wilson (Eds.), The Mountain Pine Beetle: A Synthesis of Biology, Management, and Impacts on Lodgepole Pine, Natural Resources Canada, Canadian Forest Service, Pacific Forestry Centre, Victoria, BC, Canada, pp. 304.

Stuart, J.D. (1984). Hazard rating of lodgepole pine stands to mountain pine beetle outbreaks in southcentral Oregon. Can. J. Forest Res., 14, 666-671.

Taylor, S.W. and Carroll, A.L. (2004). Disturbance, forest age, and mountain pine beetle outbreak dynamics in BC: A historical perspective, in T.L. Shore, J.E. Brooks and J.E. Stone (Eds.), Mountain Pine Beetle Symposium: Challenges and Solutions, Natural Resources Canada, Canadian Forest Service, Pacific Forestry Centre, Victoria, BC, Information Report BC-X-399, 
pp. 298.

Turchin, P. and Theony, W.T. (1993). Quantifying dispersal of southern pine beetles with mark-recapture experiments and a diffusion model. Ecol. Appl., 3, 187-198.

Waring, R.H. and Pitman, G.B. (1980). A Simple Model of Host Resistance to Bark Beetles, Forest Research Laboratory Research Note 65, Oregon State University, School of Forestry, OR.

Westfall, J. (2006). 2005 Summary of Forest Health Conditions in British Columbia, British Columbia Ministry of Forests and
Range, Forest Practices Branch, Victoria, BC, Pest Management Report Number 15, pp. 50.

Wulder, M.A., Dymond, C.C., White, J.C., Leckie, D.G. and Carroll, A.L. (2006a). Surveying mountain pine beetle damage of forests: A review of remote sensing opportunities. For. Ecol. Manage., $221,27-41$.

Wulder, M.A., White, J.C., Bentz, B.J. and Ebata, T. (2006b). Augmenting the existing survey hierarchy for mountain pine beetle red-attack damage with satellite remotely sensed data. For. Chronicle, 82, 187-202. 